\title{
EDUCAÇÃO EM SAÚDE NO PROGRAMA DE CONTROLE DA HANSENÍASE: A VIVÊNCIA DA EQUIPE MULTIPROFISSIONAL
}

\section{Health education in the leprosy control program: the experience of the multidisciplinary team}

\section{Educación en salud en el programa de control de la lepra: experiencia del equipo multidisciplinario}

\author{
Maria Cristina Dias Silva ${ }^{1}$
}

Elisabete Pimenta Araújo Paz²

\begin{abstract}
RESUMO
Esta investigação aborda a vivência dos profissionais de serviços básicos de saúde do município do Rio de Janeiro, que realizam atividades de educação em saúde no Programa de Controle da Hanseníase. Os objetivos foram compreender o significado dessas atividades e discutir como o conceito de educação em saúde se relaciona com esta prática. Utilizou-se a fenomenologia de Martin Heidegger como método de obtenção e análise dos depoimentos. Os significados evidenciaram as atividades educativas fundadas nas normas do Programa de Controle da Hanseníase e na tradição de que educação em saúde é transmitir informações necessárias ao cuidado e adesão ao tratamento. Mostrou-se um modo impessoal de desenvolver os conteúdos educativos e um discurso oriundo da prática educativa tradicional. Concluímos que a realização das atividades de educação em saúde ainda está dominada por um fazer inautêntico, mas que este não retira dos profissionais a possibilidade de se voltarem autenticamente ao ser com hanseníase.
\end{abstract}

Palavras-chave: Educação em Saúde. Hanseníase. Atenção Primária à Saúde.

\begin{abstract}
The present research addresses the experience of professionals of basic health services that perform health education activities in the Leprosy Control Program in Rio de Janeiro. The main objective of the study was to understand the meaning of these activities and to discuss how the concept of health education is related to this practice. The phenomenology of Martin Heidegger was the method used to obtain and analyze the evidence. The meanings revealed that the educational activities are based on the standards and rules of the leprosy control program as well as on the traditional concept that health education must transmit information necessary to provide care and ensure treatment adherence. This proved to be an impersonal way to develop educational contents. Plus it evidences the use of a speech that originates in the traditional educational practices. We conclude that the fulfillment of health education activities is still dominated by a non authentic practice, but this does mean that professionals are excluded from having an opportunity to truly turn towards those affected by leprosy.
\end{abstract}

Keywords: Health Education. Leprosy. Primary Health Care

\section{Resumen}

Esta investigación aborda la experiencia vivenciada por los profesionales de servicios básicos de salud del municipio de Rio de Janeiro, que realizan actividades de educación para la salud en el Programa de Control de Lepra. Tuvo como objetivos comprender el significado de estas actividades y entender como el concepto de educación para la salud se relaciona con esta práctica. La fenomenología de Martin Heidegger fue utilizada para obtener los datos y analizar los testimonios. Los significados evidenciaron que las actividades educativas están fundadas en las normas del Programa de Control de Lepra y en la tradición de que la educación para la salud es transmitir la información necesaria para el cuidado y adherencia al tratamiento. Demostro ser un modo impersonal de desarrollar los contenidos educativos y un discurso proveniente de la práctica educativa tradicional. Concluimos que la realización de las actividades de educación para la salud, permanece dominada por una práctica nada auténtica, pero esto no priva a los profesionales la oportunidad de volcarse auténticamente al ser con lepra.

Palabras Claves: Educación en Salud. Lepra. Atención Primaria de Salud

${ }^{1}$ Enfermeira da Gerência de Dermatologia Sanitária, Secretaria Municipal de Saúde do Rio de Janeiro. Especialista em Saúde Pública. Mestre em Enfermagem. EEAN/UFRJ. Brasil. E-mail: mariacrisdias@uol.com.br 2 Doutora em Enfermagem. Professora Adjunta IV. Depto Enfermagem de Saúde Pública EEAN/UFRJ. Pesquisadora do Núcleo de Pesquisa de Enfermagem de Saúde Pública. EEAN/UFRJ. Brasil. E-mail: bete.paz@gmail.com 


\section{INTRODUCÃO}

A hanseníase é um antigo problema de saúde pública no Brasil e representa ainda um dos mais importantes desafios para as autoridades sanitárias. Além de contar com agravantes inerentes às doenças de origem socioeconômica e cultural, também é marcada pela repercussão psicológica advindas das deformidades e incapacidades físicas frequentes no processo do adoecimento.

Trata-se de uma doença infectocontagiosa, crônica, causada pelo Mycobacterium leprae, uma bactéria intracelular obrigatória que compromete, principalmente, a pele e os nervos periféricos, o que pode ocasionar alteração da sensibilidade das áreas afetadas pela presença do bacilo. Esse tropismo neural é responsável pelo potencial incapacitante da doença, que, sem intervenção, gera deformidades e incapacidades nos olhos, nas mãos e nos pés. ${ }^{2}$

Os sinais e sintomas da doença são bem definidos, e o tratamento é disponibilizado aos portadores pelos serviços de saúde, notadamente as unidades de atenção primária, e a hanseníase tem cura. Apesar da tecnologia assistencial disponível, no ano de 2006 um contingente importante de pessoas foi diagnosticado já apresentando graus de incapacidade l e ll, em consequência do diagnóstico tardio. ${ }^{3}$

No Brasil, até a década de 70, a hanseníase era denominada lepra, termo relacionado a diferentes lesões corporais associadas à punição divina, decorrente de um grave pecado ou ofensa a Deus, o que gerava o afastamento até a exclusão dos doentes pelos membros da sociedade. Esta exclusão fez com que os doentes escondessem sua condição e até os dias atuais a palavra lepra carrega a conotação de que 0 indivíduo tem um problema de saúde que causa transtornos ao convívio social. Ainda hoje, a necessidade de se proteger da exclusão social, faz com que os doentes se afastem de suas atividades sociais comuns e até mesmo de familiares, com consequentes prejuízos ao tratamento adequado dos casos.

Para vencer a dificuldade em conseguir a adesão dos doentes ao tratamento e erradicar o preconceito social relacionado à lepra, o Ministério da Saúde ${ }^{4}$ pela Portaria ${ }^{0}$ 65/Bsb, de 14 de maio de 1976, proscreveu o emprego do termo lepra e seus derivados e determinou a utilização da terminologia oficial hanseníase em todo território nacional, mas até os dias de hoje a associação do nome lepra traz dificuldades ao manejo da doença por parte dos indivíduos acometidos e população em geral, que ainda a associa a pessoas com deformidades físicas.

A análise epidemiológica da endemia hansênica no Brasil, no início do século XXI, evidencia a tendência de estabilização dos coeficientes de detecção no Brasil, mas estes se apresentam ainda em patamares muito altos nas regiões Norte, Centro-oeste e Nordeste. Os esforços atuais do Ministério da Saúde têm como foco a redução da doença em menores de quinze anos, mas dificuldades para o êxito das ações estão relacionadas à existência de regiões com aglomerações de casos e indícios de trasmissão ativa. ${ }^{5}$

No que se refere ao município do Rio de Janeiro, nas últimas duas décadas, ocorreram importantes conquistas, como a expansão das atividades de controle, 0 aumento das taxas de cura, a adequação da atenção às incapacidades em nível secundário e o envolvimento de um maior número de profissionais da rede básica. Contudo, há persistência de áreas com altos coeficientes de deteç̧ão. Esses são os indicadores que devem conferir visibilidade ao problema da hanseníase no município, para que as ações de controle possam ser sustentadas e ampliadas. ${ }^{6}$

\section{Atividade educativa em hanseníase no município do Rio de Janeiro}

A partir de 1991, a ação da Gerência de Dermatologia Sanitária (GDS) objetivou, entre outras aç̃̃es, a reestruturação do Programa de Controle da Hanseníase (PCH). ${ }^{7}$ No âmbito das atividades educativas direcionadas à população visando à prevenção e ao controle da hanseníase, a Gerência intensificou a capacitação das equipes e passou a contar com a participação de outras secretarias do município, como a Secretaria de Desenvolvimento Social, e organizaç̃os sociais que trabalham com as questões envolvendo as pessoas atingidas pela doença.

0 desafio de intensificar as atividades de comunicação e educação permanece para a GDS, na medida em que no cotidiano do trabalho das equipes ainda há o predomínio da visão assistencial, em detrimento da oferta e da qualidade das ações preventivas na rede básica. ${ }^{8}$ Destacam-se especialmente neste cotidiano, as ações relacionadas à educação em saúde em hanseníase com vistas ao diagnóstico precoce, ao tratamento oportuno e à redução do preconceito social.

A despeito dos esforços visando ao esclarecimento da população sobre a hanseníase, são ainda frequentes nos serviços assistenciais a subutilização de materiais audiovisuais disponibilizados pela GDS, a falta de iniciativa para manter 0 suprimento de fôlderes/cartazes nas Unidades de Saúde e comunidades e o planejamento insatisfatório das ações de educação em saúde, nos âmbitos local e regional. ${ }^{8}$ Também a desmotivação dos profissionais em levar a termo atividades regulares de educação em saúde à população como forma de esclarecimento, e de apoio aos indivíduos em tratamento, seus contatos e familiares, continua sendo um problema de difícil resolução por parte da GDS. Para compreender como os profissionais de equipes de saúde que atuam no Programa de 
de Controle de Hanseníase em unidades básicas do município do Rio de Janeiro significam a realização das atividades de educação em saúde, desenvolveu-se esta investigação, sobre suas vivências na realização de ações de educação em saúde nesse Programa. Os objetivos estabelecidos foram compreender como a equipe multiprofissional significa a realização das atividades de educação em saúde na rede básica de saúde do município do Rio de Janeiro e discutir como o conceito de educação em saúde se relaciona com a prática destas atividades.

\section{MÉTODO}

Esta foi uma investigação qualitativa de natureza fenomenológica uma vez que se buscou compreender a vivência dos profissionais de saúde no que se refere à prática de atividades educativas no âmbito da atenção à hanseníase. $\mathrm{A}$ fenomenologia opera por meio do método que investiga a experiência, no sentido de compreendê-la, e não de explicá-la. Para a fenomenologia, importa ver o fenômeno da forma como ele se mostra na experiência vivida pelos sujeitos, os significados que os fenômenos têm para quem os vivencia e que sentidos tais significados apontam no existir humano.

É importante, ao utilizar o método fenomenológico, que o pesquisador se dirija àquilo que busca conhecer, evitando definições prévias ou conceitos teóricos que definem o fenômeno e o enquadram em explicações, que impedem que as coisas possam ser vistas em seu modo próprio de aparecer. Trata-se da epoché, o primeiro movimento do método fenomenológico. Em seguida procede-se a descrição do que já foi compreendido pelo sujeito. Por fim, realizam-se a análise e a interpretação do que foi descrito, concluindo-se a hermenêutica, ou seja, explicitando os significados que tomam sentido na totalidade da descrição que foi interpretada de modo rigoroso pelo pesquisador, considerando a cultura e a historicidade. ${ }^{9}$

Participaram da investigação 14 profissionais de diferentes categorias, sendo 7 enfermeiros, 3 terapeutas ocupacionais, 2 auxiliares de enfermagem, 2 agentes comunitários de saúde lotados em seis unidades básicas de saúde, situadas em áreas de maior prevalência da doença no município do Rio de Janeiro que realizam atividades de educação em saúde no Programa de Controle da Hanseníase. A pesquisa foi aprovada pelo Comitê de Ética em Pesquisa da Secretaria Municipal de Saúde do Rio de Janeiro em maio de 2007, com o Parecer 53A/2007 de 16.05.2007.

Realizou-se entrevista semiestruturada com os profissionais após apresentação da pesquisa e seus objetivos à chefia das unidades selecionadas e consequente aprovação para seu desenvolvimento. Os profissionais foram convidados a participar da pesquisa, e, aos que concordaram, agendou-se dia e horário na própria Unidade de Saúde para a entrevista.
Utilizou-se roteiro com questões que abordaram a vivência das atividades de educação em saúde, deixando que os sujeitos se expressassem livremente. Para evitar perda dos conteúdos das entrevistas, estas foram gravadas em meio magnético. Apresentou-se o Termo de Consentimento Livre e Esclarecido para Participação na Pesquisa aos profissionais e as entrevistas só tiveram início após sua assinatura.

A análise constou de duas etapas: a primeira foi organizar no conjunto de respostas os trechos que apresentavam características comuns aos participantes, o que resultou na construção das Unidades de Significação. Tais Unidades visaram identificar o significado expresso pelos profissionais às questões formuladas, constituindo-se em uma compreensão ainda vaga do que fazem, sem que o sentido de suas ações se mostre de maneira clara. Trata-se de uma compreensão mais imediata do que foi questionado pelo pesquisador ao profissional.

conteúdos das entrevistas, estas foram gravadas em meio magnético. Apresentou-se o Termo de Consentimento Livre e Esclarecido para Participação na Pesquisa aos profissionais e as entrevistas só tiveram início após sua assinatura.

A análise constou de duas etapas: a primeira foi organizar no conjunto de respostas os trechos que apresentavam características comuns aos participantes, o que resultou na construção das Unidades de Significação. Tais Unidades visaram identificar o significado expresso pelos profissionais às questões formuladas, constituindo-se em uma compreensão ainda vaga do que fazem, sem que o sentido de suas ações se mostre de maneira clara. Trata-se de uma compreensão mais imediata do que foi questionado pelo pesquisador ao profissional.

0 segundo e último passo da análise foi a elaboração da hermenêutica fundamentada em alguns conceitos do pensamento de Martin Heidegger como ocupação, compreensão, interpretação, discurso, linguagem e inautenticidade, presentes em sua obra Ser e Tempo. ${ }^{10}$ Na hermenêutica, o pesquisador busca evidenciar os aspectos essenciais do fenômeno, o que permite que se compreenda como se estrutura a realidade interrogada

\section{RESULTADOS}

\section{As Unidades de Significação}

Unidade 1: Para os profissionais, a educação em saúde significa transmitir informações necessárias à população sobre hanseníase.

Educação em saúde é informação que você adquire sobre determinada doença ou alguma coisa [...]. Ou transmiti-la da maneira que você aprendeu, que você viu [...]. Passar adiante (E1). 
Educação em saúde seria, no caso, passar informações [...]. As orientações que ele vai precisar para lidar com isso, e essa troca de informações. A gente vai colher com eles também as necessidades deles, o que eles necessitam, as dificuldades que eles enfrentam e a gente poder informar tudo a respeito do porquê desse estigma da doença [...] (E8).

Todas as atividades voltadas para o esclarecimento do sujeito, do cidadão, do profissional. Qualquer coisa que a gente faça: uma palestra, uma atividade educativa, um grupo, uma conversa informal que seja é educação em saúde (E9).

Esta unidade mostra que, para os profissionais, a educação em saúde é uma atividade centrada na transmissão de informações que julgam necessárias aos portadores de hanseníase e à população como um todo. Tais informações não devem deixar de ser difundidas nos espaços de saúde, reproduzindo um modo passivo de relacionamento da população com o serviço. Nos trechos acima, se destaca o modelo de uma educação tradicional, onde há um conteúdo ou conhecimento que se quer difundir a uma determinada população. ${ }^{11}$

A participação dos usuários dos serviços em atividades educativas é essencial, pois estas são mais um espaço de esclarecimento, além das consultas individuais. 0 profissional de saúde considera que deve informar o doente, os comunicantes sobre a hanseníase e que esta ação auxilia a comunidade. Crê que, quanto mais informação, melhor será o enfrentamento da doença. 0 senso comum de que educar em saúde é informar com o máximo de conteúdo domina o ambiente profissional no modo ôntico de ser no espaço dos serviços, ou seja, no modo habitual de vivenciar a ação do profissional, ou como as coisas acontecem nestes locais.

Porque apesar de a gente orientar, a hanseníase ainda é uma doença que tem alguns preconceitos como a tuberculose, as duas são doenças muito parecidas em nível de preconceito. Então, é uma coisa que eu gostaria de fazer, é um grupo específico para as famílias. Para que eles ajudem o seu familiar em casa e que não tenham os preconceitos de estar separando talher, de estar separando outras coisas (E10).

Tem que colocar o vídeo e fazer essa discussão com o paciente, até para ouvir a opinião deles, as colocações deles para você desmitificar o preconceito [...]. Eu acho que é tudo, porque tem essa troca com o paciente de saber o que ele tem como proceder, como lidar com a doença, como estigma, com o próprio tratamento, e as reações dentro de hanseníase (E12).
Os profissionais, ao realizarem atividades educativas no Programa de Controle da Hanseníase, visam à redução do preconceito que os portadores da doença podem sentir. Esta é uma questão central no trabalho que desenvolvem. Os profissionais sentem, nos grupos que organizam, como 0 preconceito sobre a doença acrescenta mais sofrimento à pessoa com hanseníase. Sabem da força impactante que existe na associação de hanseníase com lepra e todo o significado que a palavra traz, pois as imagens da lepra reportam a um quadro de isolamento, segregação e mutilação, que faz com que os portadores ou doentes tenham medo da exclusão social, pois eles sabem que a doença é contagiosa e ainda pouco conhecida pela sociedade de modo geral..$^{12}$

A hanseníase, ao ser comparada com a lepra dos tempos antigos, diminui a pessoa na sociedade. 0 profissional está preocupado em não deixar que o preconceito gerado pela palavra lepra, e que leva ao afastamento social, prejudique o tratamento e a vida familiar dos portadores de hanseníase. Ao realizar a atividade educativa, lança mão de estratégias e recursos pedagógicos que possam dar segurança para o portador e a família vencerem seus medos. Esta preocupação guia seu trabalho junto aos grupos.

\section{DISCUSSÃO DOS RESULTADOS}

\section{A Interpretação Analítica}

Nesta segunda fase da analítica, fundamentada na fenomenologia heideggeriana, ${ }^{10}$ apresentam-se as seguintes unidades:

\section{A impessoalidade no modo de realizar as atividades de educação em saúde no Programa de Controle da Hanseníase.}

Os profissionais de saúde mostraram que na maneira pública de estar nos serviços, realizando atividades educativas, há o predomínio de um modo impessoal de relacionar-se com os portadores de hanseníase e com a população em geral. Este modo de estar nos espaços assistenciais também foi discutido por outros autores ao colocarem que enfermeiros e demais profissionais de saúde desenvolvem uma prática educativa com usuários e famílias em que a multidimensionalidade do processo de adoecer não é priorizada. ${ }^{13,14}$

Os resultados encontrados evidenciaram que os conteúdos educativos ligados ao Programa de Controle da Hanseníase, que estão alicerçados em informações técnicas, são pouco trabalhados na experiência singular dos participantes, o que não contribui para um pensar crítico da realidade social na qual os sujeitos estão inseridos, e, consequentemente, o processo educativo fica prejudicado, pois os usuários deixam de se beneficiar de um importante trabalho para o alcance dos objetivos do Programa. 
A equipe de saúde se envolve com as ações educativas no modo da tradição tecnicista, que lhe dá o poder de determinar o que os portadores da doença devem seguir, a partir de orientações normatizadas pelo discurso científico contido nos manuais e guias técnicos dos programas de saúde. Este modo de ser e estar nos serviços encontra no pensamento heideggeriano a seguinte significação:

A pré-sença cotidiana retira a interpretação préontológica de seu ser do modo de ser mais imediato do impessoal. A interpretação ontológica segue inicialmente esta tendência e entende a pré-sença a partir do mundo, onde a encontra como ente intramundano $[. . .]^{15: 183}$

Os profissionais precisam instruir os portadores da doença e a população em geral sobre o problema da hanseníase. Consideram-se responsáveis por melhorar o conhecimento da população, não apenas dos que estão em tratamento, mas daqueles que convivem ou até mesmo não conseguem conviver com os portadores, pelo medo que a hanseníase provoca. Compreendem as atividades educativas como fundamentais ao processo terapêutico no plano individual. Também as compreendem como necessárias no PCH com vistas à eliminação da hanseníase como problema de saúde pública no plano coletivo.

Os profissionais neste estudo se mostraram distantes de compreender estas atividades como possíveis de fomentar a autonomia dos usuários no cuidado de si, a partir de um movimento baseado em uma prática dialógica, em que ambos podem trocar, compartilhar saberes e experiências, movimento essencial quando se pensa nas atividades educativas como um dos elementos capazes de contribuir com a cidadania dos indivíduos. ${ }^{16}$

0 profissional se empenha em cumprir da melhor forma que pode, com os recursos que estão disponíveis, aquilo que crê ser o melhor para os portadores da doença, os comunicantes e a população em geral, pois também se preocupa com o viver dos hansenianos e seus familiares. Porém, sem uma avaliação pelos serviços do impacto destas práticas junto às comunidades, o que é feito fica sem acompanhamento, e, portanto sem retorno do alcance das atividades educativas. Ao apresentar a reflexão sobre o quem da pré-sença na convivência cotidiana, Heidegger diz:
Nas ocupações do que se faz com, contra ou a favor dos outros, sempre se cuida de uma diferença com os outros, seja apenas para nivelar as diferenças, seja para a pré-sença, estando aquém dos outros, esforçar-se por chegar até eles, seja ainda para a pré-sença, na precedência sobre os outros, quer subjugá-los. Embora sem o perceber, a convivência é inquietada pelo cuidado em estabelecer este intervalo ${ }^{15: 178}[\ldots]$

Ao se compreender nas ocupações que desenvolve, 0 ser-aí-profissional se distancia dos portadores de hanseníase, seus contatos e se aproxima das normas públicas, por determinar, a partir do que ele não é, o que os portadores de hanseníase, comunicantes e população em geral têm de ser.

\section{0 falatório que domina o discurso da atividade educativa}

É na articulação do discurso educativo que se realiza nos serviços que atendem os doentes com hanseníase, que podemos ver a compreensão dos profissionais de seu modo de ser-no-mundo. Heidegger nos diz que:

O discurso é articulação "significativa" da compreensibilidade do ser no mundo, a que pertence o ser-com, e que já sempre se mantém num determinado modo de convivência ocupacional. Essa convivência é discursiva, tanto ao dizer sim quanto a dizer não, tanto provocando quanto avisando, tanto pronunciando, recuperando ou propiciando, e ainda "emitindo proposições" ou fazendo "discursos" 10:220.

Quando se empenha em esclarecer os conteúdos que são necessários para ampliar o conhecimento dos usuários sobre hanseníase ao realizar as ações educativas, o profissional está se movendo em uma fala centrada na tradição do trabalho em saúde, que deve ser capaz de prevenir a doença, promover a saúde e educar a população para a manutenção da mesma. Nas atividades de educação em saúde, os profissionais comunicam as medidas para a prevenção da doença, o modo de se adoecer, o tratamento medicamentoso e outros temas que mantém o ente dos usuários das unidades de saúde em uma disponibilidade para as metas e objetivos estabelecidos. 0 profissional assume para si esta responsabilidade e, ao assumi-la, mostra o fechamento do modo de ser ocupado. 


\section{CONCLUSÕES}

\section{O Sentido das Atividades de Educação em Saúde}

Os resultados obtidos evidenciaram que as atividades de educação em saúde no Programa de Controle de Hanseníase ainda se apresentam pouco sistematizadas e direcionadas ao processo do adoecimento, à adesão terapêutica, e não propriamente às pessoas, suas necessidades e sua autonomia. A atividade de educação em saúde para o grupo estudado ainda não atravessou a fronteira entre o saber teórico e as dimensões práticas dos que estão vivendo o adoecimento pela hanseníase.

0 profissional passa adiante aquilo que apreendeu ao longo do tempo, o que conhece a partir do que ouviu em treinamentos técnicos e cursos, mas não sabe qual o alcance de sua fala. 0 falatório técnico rouba do profissional a autenticidade do discurso que ele tanto valoriza como necessário aos usuários cadastrados no Programa, ou as pessoas que de alguma forma com eles têm relação. Como atividade recomendada pelo $\mathrm{PCH}$, a educação em saúde não dispõe de um valor próprio.

A prática educativa no $\mathrm{PCH}$ também mostrou o que Gadamer ${ }^{17}$ destacou ao analisar as questões de saúde, medicina e ciência, "(...) parte de um tratamento é o diálogo", e, sendo assim, para que este se estabeleça, é mister que haja interesse pelo que o usuário coloca e vice-versa, pois o diálogo favorece o estabelecimento das relações entre a população e os profissionais dos serviços de saúde.

Percebemos nesta investigação que a direção da atividade educativa parte do que o profissional julga que 0 outro deve conhecer para se proteger, se cuidar, se prevenir e, principalmente, aderir à proposta terapêutica. Não estamos tratando aqui de desmerecer esta prática. Sabemos que não seria de outro modo, pois o discurso da saúde é recomendação, é orientação para, é falar sempre de coisas que se julga necessário saber. Heidegger, ao discorrer sobre o falatório que prende o Ser na cotidianidade, nos traz que:

[...] repetindo e, passando adiante a fala, potencializa-se a falta de solidez, nisso se constitui o falatório. O falatório não se restringe apenas à repetição oral da fala, mas se expande no que escreve enquanto o "escritório". Aqui, a repetição da fala não se funda tanto no ouvir dizer. Ela se alimenta do que se lêê:2228.

A investigação fenomenológica sobre a prática das atividades de educação em saúde no PCH em unidades básicas hanseníase, os contatos ou a população em geral. Executar atividades de educação em saúde no modelo de uma educação tradicional nada mais é do que manter-se em uma superficial preocupação com os objetivos do Programa de Controle.

De modo geral, a preocupação com os aspectos técnicos que fazem parte das mensagens educativas, aliada ao contexto da multiplicidade de tarefas que se devem desempenhar nos serviços, não favorece um planejamento participativo com a comunidade, sobre como estas ações podem transformar o panorama epidemiológico da doença nas áreas adstritas às Unidades de Saúde. Isto não quer dizer que os resultados não sejam importantes, mas que o conhecimento que se almeja estar socializado não foi construído a partir de uma reflexão permanente sobre a ação dos profissionais.

0 desafio central para as atividades de educação em saúde não está no aprimoramento de técnicas de transmissão de mensagens, de persuasão ou sedução comunicativa nos moldes das estratégias de marketing comercial, mas em rever o pressuposto de que a existência de elementos de informação cientifica nas mensagens recebidas é suficiente para aumentar a competência e/ou a liberdade de decisão para incorporar na vida diária, o comportamento "adequado" ou desejado à sua autonomia para a manutenção da saúde ou do cuidado de si.

Esta investigação não pretendeu avaliar quais os impactos das atividades educativas no Programa de Controle da Hanseníase em Unidades Básicas de Saúde na vida dos usuários de serviços básicos de saúde, porém cabe pesquisar, em trabalhos futuros, de que maneira a população se beneficia destas práticas e o quanto as mesmas respondem às suas necessidades.

Para vencer o desafio de tornar as atividades de educação em saúde mais efetivas, inclusive para os enfermeiros que se colocam diretamente realizando esta prática, é necessário que tais atividades estejam fundadas na pré-ocupação com o outro, favorecendo o cuidado e não o direcionando, mas respeitando o ex-sistir do Ser humano. É preciso estar atento aos diferentes sentidos que a experiência da hanseníase toma na vida das pessoas e compreender que a atividade de educação em saúde é parte da mesma interface que liga o profissional à clientela.

Cabe também considerar que as ações de educação em saúde fazem parte de um processo político que requer dos profissionais o desenvolvimento de uma consciência críticoreflexiva, o que pressupõe optar por uma pedagogia que favoreça a população atribuir significado a seus problemas, a partir de elementos da sua realidade, articulando-os ao já conhecido em suas histórias de vida e, democraticamente, decidir sobre 0 cuidar de si, de sua família e da coletividade, transcendendo à 
atitude passiva baseada na ideia de que o conhecimento se dá unilateralmente e sem conflitos.

Vale ressaltar que as ações de educação em saúde não podem estar apenas na responsabilidade dos profissionais. Como processo pedagógico há também que se rever o papel das unidades de ensino que preparam os profissionais para esta prática. É importante também que a política de formação dos profissionais para o SUS possa desenvolver com criatividade o potencial daqueles que, a despeito das dificuldades que se apresentam no dia-a-dia, continuam investindo nesta área, compreendendo a educação em saúde como um campo interdisciplinar.

Este estudo buscou compreender o fenômeno das atividades de educação em saúde no Programa de Controle da Hanseníase realizadas nos serviços de atenção básica, já que estas ações estão na maior parte das vezes situadas nos profissionais. 0 conhecimento que ele trouxe, na perspectiva fenomenológica, pode servir de subsídio para que os envolvidos com o controle da hanseníase, em todos os níveis de atenção, discutam estratégias factíveis de serem incorporadas aos serviços e comunidades, para melhor qualificar a atenção à saúde.

\section{REFERÊNCIAS}

1. Ministério da Saúde (BR). Secretaria de Vigilância em Saúde. Departamento de Vigilância Epidemiológica. Manual de prevenção de incapacidades.

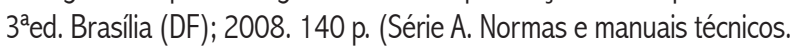
Cadernos de prevenção e reabilitação em hanseníase, 1)

2. Ministério da Saúde (BR). Secretaria de Vigilância em Saúde. Departamento de Vigilância Epidemiológica. Manual de condutas para alterações oculares em hanseníase $2^{\mathrm{a} e d .}$ Brasília (DF); 2008. 92 p. (Série A. Normas e manuais técnicos. Cadernos de prevenção e reabilitação em hanseníase, 3 )

3. Ministério da Saúde (BR). Secretaria de Vigilância em Saúde. Departamento de Vigilância Epidemiológica. Manual de condutas para Tratamento de úlceras em hanseníase e diabetes $2^{\mathrm{a}}$ ed. Brasilia (DF); 2008. 92 p. (Série A. Normas e manuais técnicos. Cadernos de prevenção e reabilitação em hanseníase, 2)

4. Ministério da Saúde (BR). Portaria nº 165, de 14 de maio de 1976. Instruções para execução das normas de controle da hanseníase, que estabelece a política de controle da hanseníase. Brasília (DF); maio1977.

5. Ministério da Saúde (BR). Secretaria de Vigilância em Saúde. Departamento de Vigilância Epidemiológica. Programa de Eliminação da Hanseníase. Situação epidemiológica da hanseníase no Brasil. Inf Epidemiol Brasília (DF); 2008

6. Secretaria Municipal de Saúde (RJ). Subsecretaria de Ações e Serviços de Saúde Superintendência de Vigilância em Saúde.
Coordenação de Doenças Transmissíveis. Gerência de Dermatologia Sanitária. Programa de Controle da Hanseníase. Relatório Anual da Gerência de Dermatologia Sanitária. Rio de Janeiro (RJ); 2008. Mimeografado.

7. Secretaria Municipal de Saúde (RJ). Superintendência de Saúde Coletiva. Coordenação de Doenças Transmissíveis. Gerência de Dermatologia Sanitária. Programa de Controle e Eliminação da Hanseníase. Relatório. Rio de Janeiro (RJ); 1994. Mimeografado.

8. Secretaria Municipal de Saúde (RJ). Subsecretaria de Ações e Serviços de Saúde Superintendência de Vigilância em Saúde. Coordenação de Doenças Transmissíveis. Gerência de Dermatologia Sanitária. Programa de Controle da Hanseníase. Relatório Anual da Gerência de Dermatologia Sanitária. Rio de Janeiro (RJ); 2006. Mimeografado.

9. Martins J, Bicudo MAV. Estudos sobre existencialismo, fenomenologia e educação. $2^{\mathrm{a}}$ ed. São Paulo(SP): Centauro; 2006.

10. Heidegger M. Ser e tempo. Tradução de Márcia de Sá Cavalcante Schuback. Petrópolis(RJ): Vozes; 2002.

11. Freire P. Pedagogia do oprimido. $19^{a}$ ed. Rio de Janeiro (RJ): Paz e Terra; 1991.

12. Ferreira AMR, Souza MJ. Representações sociais de enfermeiros do Programa Saúde da Família sobre a hanseníase: uma contribuição para a enfermagem. Esc Anna Nery Rev Enferm 2004 dez; 8(3): 448-54.

13. Backes VMS, Lino MM, Prado ML, Reibnitz KS et al Competência dos enfermeiros na atuação como educador em saúde. Rev Bras Enferm 2008 nov/dez; 61(6): 858-65.

14-Boehs AE, Monticelli M, Wosny AM, Heidemann IBS et al. A interface necessária entre enfermagem, educação em saúde e o conceito de cultura. Texto\&Contexto Enferm 2007 abr/jun; 16(2): 307-14.

15. Heidegger M. Ser e tempo. Tradução de Márcia de Sá Cavalcante. Petrópolis (RJ): Vozes; 1989.

16. Traverso-Yépez M, Araújo N. Reinvindicando a subjetividade dos usuários da Rede Básica de Saúde: para uma humanização do atendimento. Cad Saude Publica 2004 jan/fev; 20(1): 80-89.

17. Gadamer HG. 0 caráter oculto da saúde. Tradução de Antonio Luiz Costa. Petrópolis (RJ): Vozes; 2006.
Recebido em 30/10/2008 Reapresentado em 04/04/2009 Aprovado em 05/10/2009 